\title{
PRATIQUE DE LA SCIENCE SOCIALE AU MAGHREB: l'approche intercontextuelle de la modernité
}

Z. Samandi*

\begin{abstract}
Resumé: L'étude pose le problème du statut scientifique de la pratique du chercheur en science sociale dans les contextes non producteurs de savoir. Comment opérer avec des formes de savoir produites ailleurs? Telle est la question que nous avons essayé de décrypter à travers les débats développés, d'une part par les sociologues arabes et, d'autre part l'approche mise en œuvre par Arkoun, Djaït et El Jabri dans leur analyse des réalités arabo-musulmanes. Le traitement de la raison tel qu'il se donne à voir à travers les démarches examinées met au jour la pertinence de la médiation contextuelle qui ne peut se réduire à un simple transfert des outils d'analyse. L'intercontextualité comme pratique est de la sorte induite par le travers de décontextualisation-recontextualisation opéré à l'intérieur du champ de la recherche. Elle est inscrite en creux dans l'exercice d'analyse et se présente à la fois comme dimension épistémologique et herméneutique.
\end{abstract}

Mots-clés: pratique de la science sociale, modernité, raison, territorialisation des concepts, intercontextualité.

Le présent propos a trait à la pratique de la science sociale telle qu'elle s'opère dans les contextes qui peuvent être dits non producteurs de savoir. La question au centre de cette réflexion est comment opérer, s'agissant de la science sociale interprétative, avec des formes de savoir qui sont produites dans d'autres contextes?

Sociólogo do Centre d'Etudes et de Recherches Economiques et Sociales de Tunis, Tunísia. Artigo recebido em 24 maio 2003; aprovado em 30 ago. 2003. 
Ce qui est en cause, par conséquent, c'est le statut scientifique de la pratique du chercheur et, par suite, les conditions de possibilité de la production du savoir. Il est évident que ce questionnement ne prend sens qu'en rapport à une épistémé qui confère à la science sociale une nature historique (ou contextuelle).

Certes, la question du statut scientifique de la pratique du chercheur en rapport avec la nature contextuelle de la science sociale n'est pas nouvelle. Elle a été au centre de la réflexion de nombreux sociologues, tant au Nord qu'au Sud. Les sociologues arabes s'en sont préoccupés dès les années soixante-dix ce qui s'est traduit par l'organisation de nombreux débats, notamment dans le cadre de l'Association Arabe de Sociologie.

De ce fait, il sera question de revisiter ces débats pour nous interroger sur la manière dont la question a alors été traitée. D'autre part, et à partir de l'éclairage que donne l'épistémologie de la science sociale, telle qu'elle se développe depuis quelques décennies, nous questionnerons la pratique de penseurs maghrébins qui ont réfléchi sur la pertinence des outils auxquels ils ont recours.

Le débat qui a eu lieu, dans les années soixante et soixantedix, sur les pratiques de recherche de l'Ethnologie et de l'Anthropologie du monde occidental sur les autres sociétés ${ }^{1}-\mathrm{P}$. Bourdieu qui avait pris part à ce débat résume le fond de la question dans sa contribution "Les conditions sociales de la production sociologique: Sociologie coloniale et décolonisation de la sociologie" - ne concerne pas de façon directe la présente réflexion. De même que nous n'intégrons pas la position particulière de l'indigène opérant de l'intérieur de l'institution universitaire occidentale - position représentée notamment par E. Saïd. Ce cas de figure relève d'une pratique des sciences sociales un peu différente en ce qu'il implique une distanciation tout à la fois par rapport aux champs scientifiques occidental et périphérique.

Ainsi notre réflexion portera sur la pratique des chercheurs et penseurs indigènes des sociétés non occidentales et sur leur réflexion sur leur propre pratique, en tant qu'elle les inscrit d'emblée dans un rapport de pouvoir avec le champ scientifique occidental. 
Notre hypothèse est que eu égard à la nature historique de la science sociale et compte tenu de sa position, le chercheur comme le penseur indigène vont opérer dans une intercontextualité interdisciplinarité, en tant que mode d'interférence de fait entre contextes différents et sciences anthroposociales voisines. C'est, en effet, au travers d'une pratique de circulation entre différentes disciplines et différents contextes historiques que s'effectue au concret la pratique des sciences sociales dans le cas de figure qui nous intéresse.

Le trait d'union qui articule les deux termes intercontextualité - interdisciplinarité signifie que notre objet n'est pas d'aborder, d'un point de vue épistémologique, ces notions pour elles-mêmes et séparément en tant qu'approche programmée et construite (cf. L'interdisciplinarité..., 1999). Mais que nous les considérons en tant qu'elles sont inscrites en creux dans la pratique de recherche anthroposociale dans le contexte de décolonisation où le chercheur, du fait de sa position dans le champ du savoir, se trouve d'emblée dans l'acte d'élaboration de la connaissance inscrit dans ce type de démarche.

Le champ de notre analyse se limite toutefois au Maghreb et se constitue essentiellement de la réflexion menée par les sociologues arabes sur leur propre pratique ainsi que de la pratique de trois penseurs maghrébins M. Arkoun, H. Djaït et M. Abed El Jabri.

Le choix des trois auteurs s'explique par la proximité relevée quant aux principales questions au centre de leur intérêt dont notamment l'accès à la modernité du monde arabe et la rénovation de sa pensée.

C'est par le biais du thème de la raison et plus précisément au travers du traitement qui en est fait que nous tenterons de débusquer la démarche mise en œuvre par chacun des trois penseurs.

Cette réflexion se développe en trois moments. Dans un premier temps nous procéderons à la mise en place du cadre théorique qui permettra de cerner les notions d'intercontextualité - 
interdisciplinarité. Ensuite nous analyserons l'intercontextualité comme espace de pensée dans sa double modalité polémique puis critique: 1) Contexte de décolonisation et intercontextualité comme débat polémique où il sera question de ce qui a été appelée la crise de la sociologie arabe et de la demande «d'indigénisation de la sociologie»; 2) Contexte post décolonisation et intercontextualité critique. Dans cette partie on s'arrêtera sur la pensée qui tente de dépasser le débat polémique, la question étant comment construire une démarche qui évite la prédétermination par le contexte producteur du savoir? Enfin dans la partie au détour d'une démarche, nous traiterons de l'intercontextualité dans son rapport avec la subjectivité de l'analyste.

\section{Intercontextualité - Interdisciplinarité, essai de définition}

Depuis plusieurs décennies une nouvelle ligne de pensée de la science sociale interprétative s'est progressivement imposée. Elle a pris forme à travers notamment les œuvres de penseurs et praticiens tels que C. Geertz, P. Bourdieu, J-C Passeron, M. Arkoun, J. Habermas, M. Foucault et M.A. El Jabri.

On peut en voir le profil si on met en perspective quatre positions. Celle de Geertz pour qui «les formes de savoir sont toujours inéluctablement locales et inséparables de leurs instruments et entourage» (Geertz, 1986, p. 8-9). Celle de Bourdieu qui souligne que le praticien est fatalement inscrit dans un champ scientifique et par suite dans un système d'intérêts et de stratégies de spéculation symbolique «l'intérêt qu' on appelle scientifique est un intérêt pour la science qui est inséparablement un intérêt du savant à la domination scientifique du champ scientifique» (Bourdieu, p. 418).

Celle de Foucault dont la pensée archéologique nous enseigne le relativisme né de la nécessité de tenir compte de l'espace du discours, c'est à dire des présupposés théoriques et conceptuels qui sous tendent les formes du savoir: «Un savoir c'est aussi le champ de coordination et de subordination des énoncés où les concepts 
apparaissent, se définissent, s'appliquent et se transforment»(Foucault, 1969, p. 238).

Enfin, celle de la conscience plus forte encore aujourd'hui de l'étroite articulation du discours avec le lieu - tel que sociologiquement défini - de son énonciation et par suite de la nécessaire, mais cependant plutôt improbable, socioanalyse. Si «nul ne parle jamais de nulle part», le chercheur devra dans sa pratique tenir compte de ce type d'interférence (Ela, 1998, p. 17). C'est dans ce sens que Bourdieu soutient : «qu'on devrait s'interdire de faire de la sociologie, et surtout la sociologie de la sociologie, sans faire préalablement ou simultanément sa propre socioanalyse (si tant est que ce soit jamais complètement faisable)». Alors que J.M. Ela, restant sur le terrain de la pratique proprement sociologique, recommande la mise en débat des paradigmes, l'interrogation systématique des outils d'analyse. C'est-à-dire enfin de compte l'exercice d'une forme d'audace et de vigilence épistémologiques qu'il résume dans sa formule «l'épistémologie de la transgression » (Ela, 1998, p. 15).

Le second trait important de cette ligne de pensée c'est qu'elle remet en cause ce qui est qualifié de «conception technologique » de cette science, c'est -à -dire conception définie à partir de disciplines aux contours arrêtés.

Pour la science sociale interprétative telle que définie par cette perspective, cette conception doit être dépassée parce qu'elle est réductrice et schématisante: «Quand ces rubriques sont prises pour une carte de la vie intellectuelle moderne, indiquant des territoires et des frontières... elles bouchent simplement la vue sur ce qui se passe vraiment là où des hommes et des femmes réfléchissent et notent le résultat de leur réflexion.»

Geertz (1986, p. 14) pousse son anticonformisme disciplinaire jusqu'à dire qu'il compare «la sorte de chose que fait un ethnographe du même genre que moi avec la sorte de chose que fait un critique du genre Trilling et en trouvant qu'elles ne sont pas tellement différentes». Ce que dit Geertz en clair c'est que la représentation 
disciplinaire réduit l'espace de compréhension de l'objet de recherche qui est toujours plus complexe. On notera au passage combien on s'éloigne avec cette perspective de l'approche empirico-positiviste qui a été celle des chercheurs maghrébins des années soixante attachés à l'étude des structures sociales.

Cette mutation dans l'approche et la conception générale de la pratique de la science sociale est intervenue à la faveur de la pénétration de cette dernière par les vues de philosophes dont notamment Heidegger, Wittgenstein, Ricœur, de critiques, mais aussi par des pensées d'envergure, telles celle de Foucault et Habermas.

En vérité, ce n'est pas seulement le caractère des sciences sociales et la pratique du chercheur qui ont changé, mais également l'idiome de l'explication sociale ainsi que le statut de l'explication et de la compréhension. En effet, «ce n'est pas seulement la théorie, la méthode ou le sujet qui change, mais tout le sens de l'entreprise » (Geertz, 1986, p. 14). Autrement dit, par-delà les paradigmes et les théories, ce sont les préconceptions inévitables pour la pensée scientifique qui ont changé ainsi que les postulats fondamentaux concernant la nature du réel et le statut de vérité de la connaissance (Duchaste \& Laberge, 1999).

Il est certes de la nature de la science sociale, dont la fonction est critique et polémique, de se remettre en cause. L'interférence agissante de l'environnement sociétal dans l'acte de construction de l'épistémé des sciences sociales fait en effet que ces dernières «ne cessent d'exercer à l'égard d'elles mêmes une reprise critique. «Elles vont de ce qu'est la représentation, mais qui est encore une représentation, si bien qu'elles cherchent moins, comme les autres sciences à se généraliser ou à se préciser, qu'à se démystifier sans arrêt » (Foucault, 1966, p. 375).

Geertz va dans le même sens quand il note l'éparpillement des cadres et la pratique du chercheur rendue irrégulière de ce fait. Pour lui nous sommes dans les « savoirs flous et la pluralisation de l'étude de la société ».

A cette étape de l'analyse, l'interférence agissante de l'environnement sociétal dans l'acte de construction de l'épistémé 
des sciences sociales confère d'emblée à la pratique du chercheur un caractère bidimensionnel. C'est d'une certaine façon une bidimensionalité pratique qui situe le chercheur dans l'exercice de son métier dans une intercontextualité - interdisciplinarité de fait.

Certes, le problème des disciplines est récurrent en sciences sociales. Différentes études sont consacrées aux modes concrets d'interférences entre sciences anthroposociales par lesquels s'élabore une connaissance.

Pour notre part, nous traiterons de la pratique du chercheur et du penseur en science sociale dans les aires non productrices de savoir, qui dans l'acte d'élaboration de la connaissance met en œuvre des mécanismes d'ajustement interdisciplinaire et intercontextuel tout à la fois.

Plus qu'une simple procédure, ce double ajustement constitue, la condition naturelle des choses en ce qu'il revêt une fonction heuristique. Dans ce cas l'intercontextualité en particulier est constitutive du sens de ce qui fait une explication de la science sociale interprétative; sachant que cette dernière se définit par le souci d'étudier le problème comme il se présente dans son contexte et à partir de sa mise en perspective avec les formes différentes qu'il prend dans d'autres traditions. Elle rend possible de la sorte d'analyser les faits comme ils se présentent dans leur contexte propre, de les y interpréter sans qu'ils soient transférés, de façon incontrôlée, dans un registre sémantique autre, rendant ainsi improbable leur saisie à partir de leur pertinence intrinsèque.

Considéré sous l'angle des spécificités culturelles, le sens sociologique des réalités est ainsi tributaire du relativisme et de la pluralisation des cadres et non d'une quelconque conception ethnoscientifique.

L'expérience du Maghreb, à cet égard, permet de distinguer deux moments, le contexte de décolonisation et celui post décolonisation. 


\section{L'intercontextualité comme espace de pensee}

Contexte de décolonisation et intercontextualité comme débat polémique: l'argument de la spécificité culturelle

Le praticien de la science sociale dans le monde arabe est d'entrée de jeu confronté à la question du statut scientifique de sa démarche du point de vue du transfert des cadres théoriques et méthodologiques que suppose sa pratique.

Ainsi que nous venons de le voir, la genèse des formes du savoir et le caractère contextuel qui en découle soulève, comme préalable, la question de la capacité heuristique des outils épistémologiques dont il a à faire usage, celle de leur pertinence et de leur efficacité opératoire.

En réalité, cette question a interpelé le chercheur dans le contexte de décolonisation à deux niveaux. Il y avait d'abord lieu de se demander si cet appareillage de pensée est à même de parler de l'intérieur des réalités qui sont les siennes, d'en reconnaître et d'en saisir le lieu de pertinence intrinsèque. La question de la spécificité culturelle était ainsi au cour du débat.

Ensuite, et au-delà de la dimension épistémologique, l'enjeu était de savoir comment le contexte de décolonisation peut devenir espace de production de savoir et le chercheur indigène lui-même producteur de savoir.

La question de la «spécificité culturelle » développe ainsi un débat en terme de « champ ».

Penser en terme de champ, dit Bourdieu (p. 420), « C'est penser l'espace de production comme un système tel que les caractéristiques de chacun des producteurs intellectuels sont définies par sa position dans ce système ». Une telle approche amène à poser la nécessité de la sociologie des conditions sociales de possibilité d'une science sociale décolonisée. On peut distinguer deux niveaux quant à ces conditions de possibilité. Celui du rapport du champ scientifique du contexte de décolonisation avec le pouvoir intellectuel central, c'est- 
à-dire avec la science métropolitaine. « De là se déduit une foule de propriétés de la production scientifique » (Idem, p. 417). Celui ensuite du passé de la science sociale, lequel passé « fait partie des obstacles épistémologiques principaux de la science sociale, dans tous les cas et, notamment, dans le cas de la science sociale d'une société récemment décolonisée » (Idem, p. 419).

C'est au tournant des années soixante dix et quatre-vingts que le débat prend une forme plus exacerbée. «La crise de la sociologie arabe », reconnue et devenue objet de préoccupation des sociologues, débouche sur la demande d' «indigénisation des sciences sociales» qui se développe autour de l'argument de la spécificité culturelle. «Crise », « demande d'indigénisation » et «argument», l'intercontextualité devient espace de débat polémique.

Certains chercheurs qui ont vécu cette crise pour en avoir été des acteurs en attribuent l'origine à la nature contextuelle de la science sociale. Elle (la crise) «était la traduction du drame vécu par les sociologues arabes dans leurs relations avec les outils d'investigation scientifiques enracinés dans les traditions culturelles occidentales» (Zghal, 2000, p. 110). On notera ici l'emploi du mot drame, mot fort s'il en est, dont la résonance semble déborder le registre scientifique de la pratique en tant qu'acte délimité et ponctuel et renvoie à l'implication du chercheur dans son statut même, c'est-à-dire dans ce par quoi il se définit, donne sens à son discours, le fonde et le légitime. Il faut rappeler à ce propos la conjoncture particulière qui était celle des sociologues arabes travaillés, en leur qualité d'intelligentia patriote, par le souci de prendre part à la construction nationale.

La spécificité culturelle: du bricolage à la demande d'indigénisation

Voyons d'abord comment la première génération de sociologues - la génération d'avant la crise - a résolu la question de son inscription dans des formes de savoir élaborées dans la métropole? Par, on peut dire, le bricolage conceptuel essentiellement. C'est-à-dire par «la pratique des définitions opératoires dans une perspective positiviste 
et structurelle qui consiste à élargir de façon inconsidérée les propriétés structurelles des concepts collectifs comme ceux de «féodalité», «bourgeoisie», «société civile» etc., sans tenir compte de la dimension culturelle qui est la leur» (Zghal, 2000, p. 110).

La principale critique qu' on peut faire à ce niveau est que cette génération se soit contentée d'effectuer ce qui est généralement désigné par le terme «bricolage». Ce dernier suppose - dans ce cas du moins - une conception instrumentalisante des outils du savoir et donc élude la question du statut de la spécificité culturelle et le traitement épistémologique qu'elle requiert.

Une telle pratique, a conduit à l'échec du principal paradigme mis en œuvre, à savoir le paradigme développementaliste.

Définissant la première génération de sociologues arabes comme des marxistes objectifs, A. Zghal note que le paradigme développementaliste est structuralo-marxiste et de ce fait a surestimé la dimension évolutionaliste et occulté complètement la dimension culturelle.

Celle-ci s'impose à la faveur de la crise et conduit à l'émergence d'une demande d'indigénisation des sciences sociales.

Deux raisons expliquent l'émergence de cette demande. D'une part, elle a trait au contexte de la décolonisation dominé alors par la science coloniale. En effet, l'ethnologie et l'orientalisme qui « étaient essentiellement centrés sur les traditions les plus résistantes au changement et fortement fascinés par le particularisme culturel des sociétés non occidentales » (Zghal, 2000, p. 98) ne pouvaient que susciter le rejet de la part des sociologues arabes. La deuxième raison a trait aux difficultés rencontrées par la première génération des sociologues arabes à opérer avec des concepts sociologiques élaborés dans le contexte occidental et donc non nécessairement à mêmes à saisir la pertinence intrinsèque de leurs réalités.

La question ici est : un savoir indigène est-il l'alternative et la réponse judicieuse au caractère contextuel de l'épistémé. 
Cette question renvoie en réalité au débat relatif au rapport du sociologue à son objet d'étude. «Les objets de recherche entretiennent toujours une relation intelligible avec le chercheur défini sociologiquement, c'est à dire par une certaine origine sociale, une certaine position dans l'université, une certaine discipline etc.» (Bourdieu, ANO??). Donc la question à ce point de l'analyse est : Est-ce qu'il y a un rapport privilégié à l'objet de recherche ? Est-ce que l'indigénéïté constitue un rapport privilégié ?

La position de Lévi-Strauss qui défend le point de vue inverse - le privilège de l'observateur étranger - ne semble pas plus pertinente. Tout le monde connaît les nombreuses études effectuées à ce propos.

En réalité, toutes les deux procèdent d'une démarche qui élude le problème épistémologique à la base, celui des conditions sociales de possibilité de la science sociale et par suite du statut scientifique de l'approche du chercheur. Dans les deux cas, la réponse ethniciste évacue ce qui est primordial, à savoir l'épaisseur épistémologique du champ scientifique constituée « des règles du discours, des règles d'exclusion constitutives de la vérité » (Habermas, 1988, p. 293).

L'alternative ne peut ainsi pas être l'indigénisation du savoir mais l'interrogation du champ scientifique et la problématisation du rapport du chercheur dans le contexte de décolonisation à ce champ.

Critiquant l'option d'indigénisation du savoir, certains sociologues arabes proposent le comparatisme comme approche pour asseoir un jugement méthodique: «La pratique systématique des recherches comparatives comme celle d'E. Gellner sur le puritanisme protestant de la seconde moitié du XVII ${ }^{\text {ème }}$ siècle... et le puritanisme musulman de la deuxième moitié du XX ${ }^{\text {ème }}$ siècle» (Zghal, 2000, p. 110).

Le sociologue Zghal effectue dans ce sens une étude sur les mouvements islamistes comparés au puritanisme protestant de la deuxième moitié du XVII ${ }^{\text {ème }}$ qui lui permet de mettre en question la thèse de Gellner relative à 1'Islam: «Le raisonnement de Gellner est un mythe qui a limité la comparaison entre les deux puritanismes aux seuls traits psychologiques communs» (Ibidem). 
Au final, sa démarche le conduit à infirmer le postulat de « la singularité exceptionnelle de l'Islam » posé par E. Gellner et de le qualifier de préjugé culturaliste. Cette conclusion est en soi intéressante parce qu'elle oriente la recherche vers l'appréhension de l'Islam et des sociétés musulmanes de manières autres qu'à travers les préjugés culturalistes et le prisme d'une tradition érigée en paradigme. Autrement dit d'inscrire ces contextes dans l'évolution et le mouvement de l'histoire universelle.

Au plan méthodologique, le mérite d'un tel comparatisme est de pousser l'investigation au-delà des caractéristiques des acteurs sociaux pour interroger le contexte socioculturel dans son ensemble et l'appréhender comme réalité dynamique.

Seulement, une démarche comparative si elle doit se limiter au seul registre de la description, ne pourra pas prendre suffisamment en compte l'impensé du discours et ses implications épistémologiques. En effet, les infrastructures et les fondements ensevelis du sens sont mis au jour par une mise en débat de l'appareillage de la pensée. Sans cela, la fonction heuristique qui devrait être la sienne ne peut être véritablement réactualisée.

A ce point de l'analyse, on peut dire que l'intercontextualité comme débat polémique s'est développée autour de l'idée de la spécificité culturelle qui a été posée un peu à la manière d'un postulat ou d'un argument, d'ordre quasi idéologique, dans le débat.

La pensée sociologique arabe n'avait alors probablement pas, pour des raisons historiques, les moyens de penser la crise qu'elle traversait et d'en tirer les implications épistémologiques.

Contexte post décolonisation et intercontextualité comme débat critique

La pensée de la brèche et le carrefour conceptuel

La pratique de la science sociale, telle qu'elle se développe depuis trois décennies environ marque une nouvelle étape qu'on peut considérer comme constituant une phase de post décolonisation. 
Elle se caractérise par un essai de dépassement du débat polémique et la construction de toute une démarche déterminée par un souci épistémologique nouveau en rapport avec la nature contextuelle des formes du savoir.

Le principe au fondement de cette démarche et qui se substitue à celui de «la spécificité culturelle » de la sociologie de la décolonisation est celui de la mise en débat des paradigmes et de la territorialisation des concepts.

La pratique d'une intercontextualité critique comme essai de dépassement de l'intercontextualité polémique est mise en œuvre par nombre de chercheurs et penseurs du monde arabe. Dans cette partie nous nous intéressons à celle de trois penseurs maghrébins M. Arkoun, H. Djaït, M. Abed El Jabri.

Notre choix procède de deux raisons. Leur appartenance à une même tradition culturelle et leur inscription dans le sillage de la problématique de la Nahdha (renaissance arabe qui s'est développée de la fin du XIX ${ }^{\text {ème }}$ jusqu'au début du $X{ }^{\text {ème }}$ siècle). La question centrale en était comment se moderniser sans s'acculturer? question que A. Laroui (1986, p. 96) résume ainsi « se moderniser, c'est se trahir, rester fidèle à soi, c'est mourir à l'histoire».

En reprenant à leur compte cette question de la modernité, ils ont tenté chacun à sa manière d'effectuer un retour sur l'histoire et la culture arabes.

Ce faisant ils contribuent au large mouvement de renouvellement de la pensée que connaît actuellement cette aire culturelle et qui, d'une façon générale, se développe autour de la même question récurrente : Comment articuler la culture arabe à la modernité? Comment articuler la pensée arabe à la pensée rationnelle?

Ainsi, notre propos ici sera de voir, sur fond de réflexion sur la modernité, le traitement du concept raison opéré par chacun des trois penseurs. 
Quel est donc le sens de cette question qui hante, comme nous venons de le voir, la pensée arabe depuis la Nahdha?

C'est certes, celui de l'identité, du rapport au passé et à la difficile assomption du présent assimilé au retard sur l'Occident. Retard qui fait que cette aire culturelle apparaît comme mise en dehors de la rationalité, de la modernité et du temps du monde. Par suite, celui du refus de démissionner devant la difficulté d'articuler le devenir, comme construction d'une histoire, à une histoire qui semble avoir été close.

Mais ce questionnement réitéré, de façon quasi obsessionnelle, dit également le refus de renoncer à penser la continuité de l'histoire et par suite de renoncer à produire le sens de cette histoire, qui est la condition même de la pensée du sens. Autrement dit, la non résignation au non sens ainsi établi et l'acharnement dans une quête de sens, elle-même par certains, donnée comme non sens. En d'autres termes, il dit le refus de l'insoutenable déchirure culturelle, ou selon la terminologie de H. Arendt, le refus de la brèche, « le gap » dit-elle.

Et, en effet, depuis la fin du XIX ${ }^{\text {ème }}$ siècle, soit depuis la Nahdha, la pensée arabe est celle de la brèche, brèche qui émerge dans le carrefour conceptuel constitué par histoire, raison et modernité.

Celui-ci se constitue des termes par lesquels est formulée la problématique centrale des réalités dans ce contexte. De même qu'il désigne le lieu où se love le sens sociologique qui est le leur depuis la rencontre - confrontation avec la modernité occidentale.

H. Djaït, historien et penseur contemporain tresse ensemble les trois termes pour établir son diagnostic de la situation quand il souligne la nécessité d'une «prise de conscience de l'unité de l'histoire et du sens de l'histoire et de la nécessité concomitante de s'abandonner au mouvement général. Celui-ci s'appelle rationalisation et modernisation... Il est évident qu'il est incapable d'apporter une alternative à la modernité, cette modernité née en Occident, définie comme une rationalité tous azimuts et qui est devenue un destin universel. Donc, pas de salut hors de la soumission à l'esprit historique » (Djaït, 1978, p. 151). 
Ainsi, histoire, raison et modernité sont non seulement ce par quoi se définit le retard du monde arabe, mais aussi l'horizon auquel achoppe la pensée dans ce contexte et qu' elle interroge en vue d'entrer sur la scène du présent et de faire l'ouverture sur l'avenir.

Notons que ce qui fait la force du lien entre ces termes, c'est qu'ils sont liés historiquement et logiquement. En effet, ils appartiennent au même cadre historique, et à la même infrastructure mentale et sémantique. Ils s'autodéterminent et s'autosignifient.

La raison c'est celle qui a prévalu dans la modernité, la modernité se définit par le rationalisme, l'espace-temps - le champ épistémique qui confère le sens des choses - de cette raison et de cette modernité c'est l'histoire post moyenâgeuse de l'Europe.

Ce triptyque enferme de la sorte la pensée dans un type déterminé d'intelligibilité, un sens sociologique univoque des réalités qui réfère à un espace-temps particulier, celui défini par ce qu'on appelle les «temps modernes».

\section{La raison: dérive et enjeu épistémologique}

Cet espace-temps a fonctionné comme cadre de pensée depuis M. Weber notamment qui affirme l'existence d'un lien interne entre modernité et rationalisme.

Ce couplage modernité-raison a induit la confiscation historique de la raison par la modernité occidentale. La raison dès lors apparaît comme l'apanage de l'Europe post moyenâgeuse et apparaît comme coïncidant avec le cartésianisme. De la sorte, non seulement la période occidentale anté moderne, mais également l'aire culturelle arabo islamique ont été mises en dehors de la rationalité, du sens de l'histoire et par suite des temps modernes.

Il en résulte le développement d'une pensée de la généalogie univoque de la raison. Devenue véritable enjeu, celle-ci cesse de coïncider avec la fonction rationnelle qui la définit: «Le postulat de la rationalité unique induit une posture intellectuelle et une position idéologique» (Ferrugia, 1999, p. 111). 
Allant dans le même sens, d'autres chercheurs dont notamment Hervé(G) et Baudry $(\mathrm{H})$ dans le National Cartésianisme engagent la réflexion sur l'héritage culturel du «mythe Descartes » et dénoncent la «mystification » qui s'est opérée à partir de Descartes sous le couvert de la rationalité. Ils mettent au jour les injonctions du dogmatisme camouflé par la « raison-foi » enfermé dans le référentiel cartésien (Herve \& Baudry, 1999).

Ce qui nous intéresse ici c'est de noter que dans le procès de son évolution, le cartésianisme a phagocyté la raison, et s'est posé comme l'unique généalogie, confondant ainsi l'histoire de la raison avec l'histoire occidentale européenne-chrétienne.

Après l'Ecole de Francfort qui a cherché à dévoiler, derrière l'illusion d'un raison universelle, le discours dominant, l'efficacité, l'expertise et une idéologie aliénante selon Marcuse (1996, p. 9), Habermas souligne à son tour la dérive de la raison.

Les sciences modernes se sont désolidarisées de la raison à laquelle avait prétendu la tradition philosophique; et, avec elles le rapport classique entre théorie et pratique s'est renversé. Les sciences de la nature qui produisent un savoir techniquement exploitable sont devenues une forme réflexive de praxis; elles sont désormais la première force productive. (Habermas, 1988, p. 90)

Avec la critique Habermasienne et celle de la pensée postmoderne d'une façon plus générale, ce n'est plus seulement la genèse de la raison assimilée au rationalisme des Lumières qui est mise en cause, mais aussi sa fonction heuristique.

Rapportée à la pratique dans les sciences sociales, cette dérive induit une posture rationalisante qui tend à supplanter la raison en tant que fonction critique et interrogative. D'autre part, elle induit le tout rationnel qui prétend réduire l'humain au rationnel. Ce qui n'est pas conforme à la raison ainsi définie, est repoussé hors des frontières de la modernité et des Lumières et est assimilé à l'archaïsme, au déclin et à la tradition.

Modernité et tradition prennent ainsi une acception particulière et se constituent en deux mondes qui se font face. Et la pensée dans 
la science sociale, devenue dichotomique, classera les contextes sociétaux en deux univers hétérogènes sinon antinomiques: «Les pères fondateurs de notre discipline (Durkheim, Weber et Marx) étaient ainsi tous des enfants des Lumières» (Wallerstein, 1999, p. 160).

Le champ de la science sociale, ainsi qu'on peut le voir, est étroitement mêlé au débat philosophique sur la raison. Rapporté à l'analyse des contextes non occidentaux notamment, ce débat se traduit par un enjeu épistémologique, celui relatif à la pertinence des approches et à la valeur heuristique des paradigmes mis en œuvre par le chercheur.

Mais pour ce qui est du contexte arabo musulman en particulier, cette posture rationaliste formelle qui réfère au postulat de la rationalité unique, a amené à ériger la tradition en paradigme au travers duquel sont appréhendées de façon privilégiée les réalités ; réalités désormais inscrites dans une distance épistémique par rapport à la rationalité et dans un rapport d'extériorité par rapport à la modernité.

C'est donc à la faveur d'un glissement sémantique, l'assimilation raison et cartésianisme que se met en place une clôture épistémologique. Ayant noté la « difficulté à transplanter ailleurs une rationalité aussi intimement mêlée à toute une tradition historique», H. Djaït se demande «y a-t-il un moyen de dépasser cette antinomie et d'accéder à une rationalité qui ne soit entachée d'aucune empreinte spécifique et qui soit véritablement universelle, extensible à l'ensemble de l'humanité» (apud Penseurs... 1993).

Pratique généalogique et territorialisation des concepts

En butte à cette clôture épistémologique et en vue de la lever, les penseurs M. Arkoun et M. Abed EL Jabri ${ }^{2}$ posent «le raison islamique » (pour le premier) et « la raison arabe» (pour le second) faisant apparaître le cartésianisme comme genèse particulière. ${ }^{3} \mathrm{La}$ territorialisation du concept raison ainsi opérée désignera dès lors autant de processus particuliers de la construction historique de la fonction rationnelle universelle. 
« Raison arabe » et « raison islamique » peuvent être dites alors filles de la culture de l'impertinence qui naît du débat avec les paradigmes dominants et de leur interrogation. J.M. Ela (1998) parle dans ce sens de «l'épistémologie de la transgression» qu'il pose comme condition pour la réactivation de la fonction heuristique des outils d'analyse, notamment quand ils sont mis en œuvre pour l' analyse des contextes autres qu'occidentaux.

Contrairement à la « spécificité culturelle » qui semble ne pas avoir prouvé sa portée heuristique, ces notions se présentent comme des concepts-débats dans ce sens où ils sont construits à partir d'une interrogation du cadre théorique et de la mise en débat de ses catégories. Leur pertinence et leur efficacité opératoire consistent dans le fait qu'ils peuvent alors parler de l'intérieur des réalités historiques et des contextes sociologiques particuliers.

C'est à la faveur d'une pratique généalogique que les notions de «raison arabe » et de «raison islamique» sont produites.

La généalogie mise en œuvre par les penseurs est celle définie par Habermas comme étant non pas ce qui doit «se mettre à la recherche d'une origine, mais aller à la découverte des commencements contingents des formations discursives» (Habermas, 1988, p. 295). Autrement dit une généalogie qui étudie la manière dont se forment les discours. Elle porte donc sur les pratiques qui sont corollaires du discours. Le but en est ainsi d'analyser la multiplicité des histoires effectives: «Il faut en finir avec une histoire au singulier... pour la dissoudre en un pluralisme d'îlots discursifs» (Idem, p. 297).

On retrouve ici l'idée de Geertz relative à l'éparpillement des cadres pour éviter ce qui pourrait ressembler à une sclérose de la connaissance dans ce domaine. Ainsi «raison arabe » et « raison islamique » portent le débat non pas sur le fait raison lui-même, mais sur la question généalogique dont le corollaire est l'affirmation d'une généalogie plurielle. Ce qui en résulte, ce n'est pas une remise en cause de l'universalité de la fonction rationnelle qui ne peut être liée au caractère supposé univoque de sa généalogie. 
En fin d'analyse la démarche intercontextuelle mise de la sorte en œuvre, est différente de la perspective ethnoscientifique puisqu'elle situe le chercheur dans un espace épistémologique critique qui, sans remettre en cause le caractère universel des catégories et concepts, tente d'en cerner le noyau temporel pour réactiver la pertinence heuristique qui est la leur et rendre possible de reconnaître les dynamiques profondes qui travaillent les formations historiques particulières. C'est en réalité un travail de décontextualisation recontextualisation nécessaire si l'on veut parler l'intérieur des réalités et les appréhender à partir des lieux de leur pertinence intrinsèque.

Pour l'analyste maghrébin, la territorialisation du concept « raison » opère une distanciation par rapport au schéma des Lumières. Autrement dit opère le découplage modernité - raison. L'espace discursif ainsi ouvert par ce découplage libère de l'espace-temps défini par la modernité occidentale. Il rend possible de ce fait l'émergence de ce que Habermas appelle un îlot discursif autre. C'est précisément à partir d'un tel lieu que se déploie la pensée de Arknoun et d'El Jabri et d'une façon plus générale la réflexion portée par le large mouvement de rénovation de la pensée arabo-musulmane.

L'intérêt épistémologique à ce niveau est qu'il devient possible d'opérer de l'intérieur du contexte historique en question, l'articulation de la pensée et de la culture à la modernité et à la rationalité.

Le dilemme tel qu'il est exprimé par A. Laroui (1986) «se moderniser c'est se trahir, rester fidèle à soi c'est mourir à l'histoire » peut être alors dépassé. De même qu'il devient possible de sortir des problématiques formulées en terme de «conciliation entre tradition et modernité » ou de la pratique d'un sommaire « bricolage » auquel a eu recours la première génération des sociologues arabes.

Raison territorialisée et fonction d'articulation

Ces penseurs réfèrent au long procès de développement de la pensée arabo musulmane qui a culminé au XII ${ }^{\text {ème }}$ siècle (ère chrétienne) 
avec la pensée d'Avérroès lequel philosophe a incarné alors « la figure de la raison » (Fabre, p. 5).

Ce retour en arrière permet de rétablir ce que $\mathrm{H}$. Arendt appelle les «médiations» entre le passé révolu et l'avenir et qui de son point de vue sont nécessaires pour comprendre la modernité. Les «médiations» en effet, assignent un passé à l'avenir et assurent une continuité dans le temps, et par suite le sens du monde et l'unité du sens. Et peut être plus simplement les conditions de la pensée du sens.

Djaït (1980) réfère à une autre inscription généalogique qui renvoie au processus de rationalisation de la vie sociale. Cette rationalisation transparaît dans la trilogie Etat-ville-monnaie à la faveur de la rupture d'avec la période païenne anté islamique (la jahilya).

L'enjeu épistémologique est que c'est par le biais de la raison ainsi réappropriée et inscrite dans le procès de l'histoire arabo musulmane qu'il devient possible de lever les clôtures épistémologiques et d'opérer de l'intérieur l'articulation de la culture arabo musulmane à la modernité. H. Arendt (1972, p. 15) souligne l'importance de l'articuler de l'intérieur pour la pensée et pour l'histoire quand elle note «... sans cet achèvement de la pensée après l'acte, sans l'articulation accomplie par le souvenir, il ne restait tout simplement aucune histoire qui pût être racontée».

On devrait pour terminer ce point, noter ici que la démarche qui a le souci d'articuler passé et présent de l'intérieur des contextes en recourant à la mise en place de « médiations » conduit à resignifier le rapport - tel que défini par la pensée positiviste - du passé et du futur, et notamment celui de la tradition et de la modernité, puisque ce dernier cesse d'être pensé en termes de rupture et d'opposition. La tradition devient, en effet, ce pourquoi il y a pensée, histoire et sens.

...Sans tradition - qui choisit et nomme, qui transmet et conserve, qui indique où les trésors se trouvent et quelle est leur valeur - il semble 
qu'aucune continuité dans le temps ne soit assignée et qu'il n'y ait, par conséquent, humainement parlant, ni passé ni futur mais seulement le devenir éternel du monde et en lui le cycle biologique des êtres vivants. (Arendt, 1972, p. 14)

Ainsi Arkoun, El Jabri et Djaït pour reconstituer la continuité de l'histoire et l'unité de sens rompent avec la pensée des Lumières. Et c'est la « raison territorialisée » qui a rendu possible l'articulation de ce qui semblait logiquement désarticulé.

Le cartésianisme, en revanche, en se posant comme genèse univoque de la raison a produit la rupture dans la continuité de l'histoire, en opposant les temps modernes au moyen âge judéochrétien. La raison cartésienne n'a pu de ce fait revêtir la fonction d'articulation dans le procès de la pensée occidentale.

La pensée critique de la modernité et d'une certaine manière, la pensée post moderne, opèrent un retour sur la genèse de la raison, pour précisément tenter de remettre en place les «médiations » et reconstituer l'unité du champ symbolique dévasté.

Pour clôturer ce point, on peut dire que la différence du point de vue de l'approche et de la pensée sociologique entre les deux moments que nous avons distingués, décolonisation et post décolonisation, réside dans le traitement de la question du rapport du chercheur indigène en science sociale au champ scientifique occidental. Dans la seconde phase, le dépassement du débat polémique a été rendu possible grâce à l'interrogation du statut scientifique de la pratique du chercheur et au traitement épistémologique de son rapport à l'épistémé occidentale.

\section{Au détour d'une démarche: le chercheur face a ses certitudes}

L'intérêt de cette partie est qu'elle a trait à une expérience particulière d'intercontextualité. Plus précisément de confrontation entre deux contextes, celui de l'analyste et celui de son objet d'étude, le mouvement des femmes islamiques, ou plus exactement l'intelligentia féminine qu'il a secrétée (Samandi, 1996). 
A un premier niveau, la question ici est celle du rapport privilégié à l'objet d'étude. C'est une situation, en effet, dans laquelle le chercheur indigène rencontre, comme n'importe quel chercheur, des difficultés à construire un rapport d'objectivation par rapport à son objet.

A un autre niveau, le mouvement des femmes islamiques peut se révéler un lieu où l'analyste de culture arabo-musulmane se risque parce que de prime abord, en tant que chercher on est interpellé dans son épaisseur subjective et émotionnelle.

En effet, dans un premier temps, le discours de ces femmes, étant différent, est reçu comme un discours pathologique.

Dans un second temps et parce qu'il fallait expliquer le paradoxe sinon la provocation intellectuelle, que constitue l'adhésion souvent massive des femmes à ce courant, le rejet fait place à la curiosité et au désir de comprendre. Une approche sociologique devenait alors envisageable. La construction d'un rapport d'objectivation devient effectivement possible dès l'instant où le chercheur est contraint de se défaire de ses préjugés et de ses choix et se sent à même de procéder à une réévaluation de son rapport à l'objet d'étude.

Peut-on toujours y parvenir de façon satisfaisante? De l'étude entreprise se dégage l'impression d'une pensée négociée, voire censurée. Une réflexion mérite d'être menée sur la signification de l'attitude du chercheur qui a ainsi bridé sa pensée face à un objet qui bouscule ses certitudes.

Un second intérêt justifie cette partie, celui relatif au traitement de la raison tel qu'il est effectué par l'intelligentia des femmes islamiques. Ce mouvement illustre ainsi un autre traitement de la raison, traitement qui consiste à inscrire cette dernière dans les textes fondateurs de l'Islam de façon à opérer de l'intérieur l'articulation de l'Islam et de la modernité. 


\section{Principaux résultats de l'étude}

L'étude en question a porté sur l'intelligentia féminine islamique dans le Maghreb et en Turquie. Quatre points s'imposent dans ce bref rappel:

$\left.1^{a}\right)$ Les femmes islamiques posent une exigence de continuité dans l'assomption de la modernité. Elles rejettent en revanche une définition du modernisme qui les inscrit dans une rupture par rapport à leur être historique et culturel.

$\left.2^{a}\right)$ Elles refusent de se couper de la référence religieuse, mais s'employant à en tirer le maximum d'avantages et à faire prévaloir une lecture moderniste des préceptes de l'Islam.

$\left.3^{a}\right)$ Leur stratégie consiste à livrer bataille dans le champ du savoir qu'elles investissent. Ainsi, elles s'approprient l'islam et développent leur propre argumentation exégétique. En s'affirmant comme sujet du savoir et de la connaissance, elles se confèrent le pouvoir de développer un discours différent qu'elles opposent tout à la fois à l'orthodoxie (masculine) et à la tradition.

Ce qu'il est intéressant de souligner à ce niveau, c'est que, pour la première fois, se trouve exprimé sur le mode de la revendication scientifique et politique, le refus de la confiscation historique de la chose religieuse par les hommes et par le pouvoir. Elles se posent de la sorte en sujet de la parole religieuse. Ce phénomène inédit n'a pas été encore analysé. Nous pensons que la portée qui est la sienne se révèlera importante au vu des retombées qu'elle aura à moyen terme. En effet, il est probable que, pour ces contextes, la problématique de la modernisation n'aura plus à référer exclusivement à l'Occident. Désormais, ces mouvements pourront conjuguer légitimement Islam et modernité.

$4^{\text {a }) ~ P a r ~ c e t t e ~ a p p r o p r i a t i o n, ~ e l l e s ~ s e ~ d o n n e n t ~ l e ~ m o y e n ~ d e ~ l a ~}$ gestion et du contrôle de la référence islamique. La connaissance, le savoir sont ainsi mobilisés comme arme en vue de leur émancipation.

En fin d'analyse, on peut dire qu'elles ont une démarche qui vise à développer une nouvelle culture islamique où la femme se 
trouve réhabilitée et abolie la distance entre islam et raison. Ce mouvement intellectuel établit ainsi un lien logique entre la levée de l'oppression que subissent les femmes et la raison. De leur point de vue en effet, seule une lecture rationnelle de l'islam est susceptible de favoriser une véritable émancipation féminine et de fonder l'égalité entre les sexes.

Ainsi l'émancipation de la femme est concomitante à l'émancipation de la culture arabo-islamique. Ces deux procès constituent les deux faces d'un seul projet. Le changement pour les femmes ne pouvant s'opérer que de l'intérieur du champ culturel et par une relecture de l'histoire. Par cette démarche, l'intelligentia féminine islamique se démarque de l'élite dont le moderniste réfère à la modernité occidentale. Il y a là, en même temps qu'une nouvelle figure de la femme émancipée et moderne une nouvelle conception de la modernité.

L'argumentation des femmes islamiques ou la raison intratextuelle

On peut dire que l'argumentation en faveur de la cause féminine telle qu' elle est développée par le mouvement religieux féminin prend appui sur ce qui est considéré comme un acte fondateur, à savoir l'interdiction faite par l'Islam d'enterrer vivantes les petites filles.

Pour cette perspective, une telle interdiction qui avère deux dimensions inhérentes à l'Islam - raison et justice - en fait une religion porteuse du salut pour les femmes opprimées, parce que porteuse de rationalité. Ainsi, le couplage des deux notions - raison et justice permet à ce mouvement d'asseoir une nouvelle lecture des préceptes religieux, lecture qui bouscule l'ordre traditionnel en préconisant la reconnaissance de l'égalité des droits entre les deux sexes. La question de la polygamie illustre le profit que les femmes islamiques tirent de ce couplage. Elles ne manquent pas de rappeler, en effet, que c'est au nom du devoir de justice à l'égard de toutes les épouses que le verset coranique ébranle la légitimité de la polygamie.

L'Islam dès lors, ne peut être considéré comme source d'oppression. Il se pose en revanche comme instrument de libération. 
Ainsi, en inscrivant la raison dans le texte sacré, les femmes islamiques inversent totalement le rapport habituellement construit entre les trois notions, Islam, raison et femmes. Elles mettent ces termes du même coté et considèrent qu'il y a un lien interne entre eux.

En effet, l'«irrationnel» qui est, selon elles, synonyme d'ignorance et réfère à la Jahilya, est anté et anti islamique. La «Jahilya» (période anté islamique) est ainsi tout à la fois anté-islam et anti-islam, anté-raison et anti-raison et par suite anti-femme.

Cette démarche leur permet d'invalider, du point de vue religieux, toute lecture «maschiste » et opprimante, dès lors que l'oppression des femmes est référée à la « Jahilya ».

La raison serait ainsi ce par quoi s'opère l'articulation de l'islam et de l'émancipation des femmes et, d'une manière plus générale, de la culture arabo-islamique et des valeurs de la modernité.

Pour ce qui est enfin du Hijab (voile religieux) qu'elles revendiquent, on peut dire que, à travers ce signe du corps et du vêtement, elles entendent donner visibilité à une nouvelle figure de la femme musulmane, cultivée et émancipée et conciliant modernité et islam.

Elles considèrent qu'il constitue un instrument de libération dans ce sens que son port leur permet de s'affirmer dans l'espace public sans être accusées de susciter le désordre et la discorde, la fonction du Hijab étant de cacher non la femme mais la féminité. C'est par cette nuance et par ce distingo établi entre les deux notions de «femme» et de «féminité», distingo qui réfère à l'opposition dudedans et du-dehors, du privé et du public, que les femmes islamiques resignifient les notions clefs - qui ont servi à justifier leur oppression et d'une façon générale l'ordre traditionnel - à savoir 'Aoura (manquement au devoir de pudeur), liberté, privé, public, fitna. $\mathrm{Ce}$ travail de resignification rompt l'association séculaire entre l'être féminin et l'ordre du-dedans et redéfinit le statut social de la femme (Samandi, 1999). 


\section{Le foulard ou la question démocratique}

L'intercontextualité ne revêt pas uniquement un statut opératoire en rapport à la pratique dans la science sociale. Ce qui, depuis quelques années, constitue en France «l'épisode du foulard islamique » en est une expression empirique qui relève de la pratique des acteurs sociaux.

En effet, à travers cette question du « foulard » et notamment à travers la diversité du sens qu'il prend et du traitement qu'il appelle d'un pays à l'autre, on voit comment la même problématique, celle de l'ordre social et de l'infraction à la règle institutionnelle, s'ordonne selon une symétrie inversée. Alors que dans des pays comme l'Iran ou l'Arabie Saoudite, la conformation à la loi suppose le port du Hijab. Dans d'autres, Tunisie, Turquie et France notamment, c'est arborer « le foulard » qui constitue l'infraction et menace l'ordre social.

Appréhendée sous l'angle de la sociologie politique, ce télescopage pose la question de l'Etat moderne dans le contexte de la mondialisation et du multiculturalisme, autrement dit le contexte post Etat-Nation. Qu'est-ce qu'un Etat neutre? Qu'est-ce qu'un Etat de droit aujourd'hui? Comment concilier ses fondements avec le principe de la démocratie communautarienne?

D'une certaine façon, le communautarisme fait émerger l'intercontextualité au sein des sociétés et des pratiques politiques nationales. C'est en vérité tout le débat qui a mis aux prises différents acteurs sociaux et politiques ainsi que les philosophes libéraux et les philosophes communautariens. Il y a là de la sorte une nouvelle forme de tension, l'intercontextualité dans l'espace public, qui interpelle le chercheur en sciences sociales.

Du point de vue de la science sociale, on peut dire que, depuis les Lumières, le questionnement a référé essentiellement à la tension entre individu et société, entre le particulier et le général. La sociologie notamment, s'est largement employée à répondre à la question comment gérer cette tension? 
Aujourd'hui, les catégories en se métissant produisent un nouveau type de tension, celui entre les différents groupes qui constituent la société pluriculturelle. La question est comment gérer cette situation inédite? Comment l'interroger dans la perspective la nouvelle ligne de pensée dans la science sociale qui refuse les paradigmes schématiques et devient antiformaliste et relativiste? Comment penser la démocratie dans la perspective communautarienne?

\section{Conclusion}

La question au point de départ de ce travail est: comment opérer dans les situations de post colonisation eu égard à l'irrépressible historicité des déterminations épistémologiques, méthodologiques et herméneutiques dans la science sociale interprétative?

En effet, l'interrogation implicite, comment m'approprier les concepts de l'autre pour penser les réalités de mon contexte ouvre directement sur la difficulté de la prise en compte des objets empruntés en tant qu'ils sont déjà l'objet d'interprétations sociales.

Cette difficulté ne peut être éludée car elle constitue ce que Geertz (1986, p.18) considère l'esprit même de la science sociale interprétative, a savoir, "la remise dans le contexte des signifiants qui est une façon plus utile de comprendre comment ils signifient et quoi, que celle qui consiste à les forcer en paradigmes schématiques ».

Notre travail se situe précisément à ce niveau. Et c'est notamment le traitement de cette difficulté que nous avons essayé de décrypter à travers les débats développés par les sociologues arabes dans les années soixante et soixante dix d'une part, et les démarches mises en œuvre par trois penseurs maghrébins, d'autre part.

$\mathrm{Au}$ terme de cette analyse, on peut dire que ce rapport d' «extériorité» avec les outils situe la pratique dans un espace d'intercontextualité de fait.

Nous avons tenté, sous l'angle de la problématique de la modernité, de cerner les différentes approches ainsi mises en œuvre. 
La première qui s'est dégagée, conception plutôt instrumentalisante des outils du savoir, a éludé la dimension épistémologique et herméneutique. Le paradigme «développementaliste» a ainsi réduit la problématique de la modernité à une expression schématisante et plutôt mécaniste, dont les sociologues arabes - conscients de ne pas avoir réussi à saisir leurs réalités dans la pertinence intrinsèque qui est la leur - en ont rapidement éprouvé les limites.

La seconde a procédé à la remise en débat des outils d'analyse. Celà a permis de développer une approche plus à même de parler de l'intérieur des réalités et d'en appréhender les dynamiques profondes en ce qu'elle prend en compte tant du point de vue épistémologique qu'herméneutique le travail de remise dans le contexte des signifiants.

Le traitement de la raison, concept central pour penser la modernité, tel qu'il transparaît à travers la démarche de H. Djaït, $\mathrm{M}$. Abeb El Jabri et M. Arkoun constitue une illustration de ce type de travail.

Chacun, à partir de l'optique qui est la sienne, a mis en œuvre une pratique intercontextuelle qui a permis de remettre dans le contexte arabo-musulman le concept de raison, d'en réactiver la fonction heuristique et par suite de rendre possible une articulation de la modernité de l'intérieur du procès d'évolution de la pensée et de l'histoire dans cette aire culturelle.

De la sorte, la médiation contextuelle, en tant que pratique, permet d'opérer non pas un simple transfert des signifiants - ce qui ne rend pas possible l'accès au sens intrinsèque des choses et à la dynamique qui travaille en profondeur les réalités - mais leur «traduction ».

La traduction est à prendre ici dans le sens de Geertz qui souligne le souci de transporter intact le sens d'un contexte à l'autre. « Traduire » ici ne veut pas dire une simple refonte de la façon dont les autres présentent les choses afin de les présenter en termes qui sont les nôtres (c'est ainsi que les choses se perdent), mais une 
démonstration de la logique de leur présentation selon nos propres manières de nous exprimer... c'est appréhender « leurs vues » dans «nos vocabulaires » (Geertz, 1986, p. 16). Autrement dit, c'est la prise en compte de la présence fatale de l'autre qui induit la nécessité de la pratique intercontextuelle.

Dans l'approche des trois penseurs, la raison a revêtu la fonction de médiation contextuelle et a été le lieu qui a révélé différents procès de rationalisation. Le principe d'une généalogie plurielle de la raison rend alors possible d'abolir les clôtures épistémologiques - qui en l'occurrence enferme la pensée dans la dichotomie tradition modernité - et par suite d'articuler de l'intérieur la modernité au procès d'évolution de l'aire arabo musulmane.

Ainsi, l'intercontextualité n' est pas à appréhender comme projet ou même comme apport et n'est donc pas à comprendre sur le modèle pragmatique d'un programme à remplir, mais comme une émergence pratique entre diverses modalités de médiation à l'intérieur de la recherche.

Tout comme l'interdisciplinarité qui n'est pas explicitement reconnue et qui s'impose dans la pratique du chercheur en tant que médiation en permanence à l'œuvre, l'intercontextualité se présente sur le modèle analytique d'une situation épistémique première à éclairer.

Inscrite en creux dans l'exercice de recherche et de réflexion, elle se présente à la fois comme dimension épistémologique et herméneutique. Ce qui y amène, c'est l'incapacité des outils tels qu'ils s'offrent au départ à rendre compte des difficultés et des paradoxes auxquels se trouvent confronté le chercheur dans le contexte de postdécolonisation.

\section{Notas}

1 Voir à ce sujet Colonna.

2 Voir à ce sujet notamment « Penseurs Maghrébins Contemporains ». 
3 Cette genèse s'est voulue en rupture avec les deux traditions gréco-latine et judéo-chrétienne. Voir à ce sujet notamment Touraine (A), Critique de la modernité... Paris: Fayard, 1992.

Abstract: This paper deals with the problem of the scientific practice of researchers in social science who use forms of knowledge historically produced in other contexts. How to operate with alien forms of knowledge? The paper tries to answer this question through the discussion of sociologists from the Arab world and through the way that Pr. Arkoun, Pr. Djait, Pr. El Jabri deal with the issue in the analysis of Moslem Arabic realities. The different approaches of the object of this paper shows how the contextual mediation is relevant to social science. The tools of analysis are not automatically transferred but they subjected to the necessity of decontextualization and recontextualization. Thus, intercontextuality as a researcher practice is a process of dealing with the concepts of social science. Intercontextuality operates within the scientific practice and has both an epistemological and an hermeneutical dimension.

Key-words: social science, modernity, reason, territorialization of concepts, intercontextuality.

Resumo: Este artigo trata do problema da prática científica de cientistas sociais que usam formas de conhecimento historicamente produzidas em outros contextos. Como lidar com estas formas de conhecimento? $\mathrm{O}$ artigo tenta responder esta questão através da análise das realidades árabe-muçulmanas realizadas, de um lado, por sociólogos árabes e de outro por autores como Arkoun, Djait e El Jabri. As diferentes abordagens do problema mostram que a mediação do contexto é relevante nas Ciências Sociais. Os instrumentos de análise não são transferidos automaticamente, mas estão sujeitos à necessidade de descontextualização e reconstextualização.

Palavras-chave: prática da ciência social, modernidade, razão, territorialização dos conceitos, intercontextualidade. 


\section{Références bibliographiques}

ARENDT, H. La crise de la culture. Paris: Gallimard, 1972.

BOURDIEU, P. Les conditions sociales de la production sociologique: sociologie coloniale et décolonisation de la sociologie. In: LE MAL de voir. Cahiers Jussieu Univ ParisVII, Publication de Colonna (F).

COLONNA, F. Le mal de voir.Cahiers Jussieu,Univ Paris VII.

DJAÏT, H. Al Koufa, naissance de la ville arabe. Paris: Maisonneuve et Larose, 1980.

DJAÏT, H. L'Europe et l'Islam. Paris: Seuil, 1978.

DUCHASTE, J.; LABERGE, D. La recherche comme espace interdisciplinaire. Revue Sociologie et Sociétés, Montréal, v. 31, n. 1, 1999.

ELA, J. M. Les sciences sociales à l'épreuve de l'Afrique: les enjeux épistémologiques de la mondialisation. In: GLOBALISATION et Sciences Sociales en Afrique, Thème 6: Epistémologies. Sénégal: Codesria, 1998.

FABRE, Th. Propos sur la raison. Qantara, n.14.

FERRUGIA, F. Une brève histoire des temps sociaux: Durkheim, Halbwachs, Gurvitch. Cahiers Intenationaux de Sociologie, Paris, v. 106, jan./ juin, 1999.

FOUCAULT, M. L'archéologie du savoir. Paris: Gallimard,1969.

FOUCAULT, M. Les mots et les choses. Paris: Gallimard,1966.

GEERTZ, C. Savoir local,savoir global. Paris: PUF. 1986.

HABERMAS, J. Les discours philosophique de la modernité. Gallimard, 1988.

HERVE, G; BAUDRY, H. La nuit des Olympica. Essai sur le nationalcartésianisme. Descartes inutile, La France cartésienne, Adieu Descartes. Paris: L'Harmattan, 1999.

L'INTERDISCIPLINARITÉ ordinaire. Revue Sociologie et Sociétés, Montréal, v. 31, n. 1, printemps 1999.

LAROUI, A. Islam et modernité. Paris: La Découverte, 1986.

MARCUSE, H. L'homme unidimensionnel. Paris: Seuil, 1996. 
PENSEURS maghrébins contemporains (collectif). Tunis: CERES Productions, 1993.

SAMANDI, Z. Difficultés de l'approche identitaire : le mouvement des femmes islamistes. In: autor? Les mouvements sociaux en Tunisie et dans l'immigration. Tunis, 1996. (Cahiers du CERES, Série Histoire, n. 6).

SAMANDI, Z. Le hijab révolutionnaire contre le voile traditionnel: le corps de la femme et l'ordre social. Revue Tunisienne de Sciences Sociales, Tunis, n. 119, 1999.

TOURAINE, A. Critique de la modernité. Paris: Fayard, 1992.

WALLERSTEIN, I. falta o título Revue Société Contemporaine, n. 33-34, janv./fév. 1999.

ZGHAL, A. Pour changer la notion de spécificité d'un objet désir en objet de recherche sociologique.In: MAHFOUD-DRAOUI.; BEN SALEM, L. Modernité et pratiques sociologiques. Tunis: Centre de Publication Universitaire, 2000. 